\title{
DECISIONS
}

\section{Lower urinary tract symptoms in a 66-year-old man}

\author{
Dean S. Elterman MD, Steven A. Kaplan MD
}

A 66-year-old man presents to his primary care physician with a six-month history of urinary urgency. He wakes three times per night to void and has noticed that his urinary stream has slowed. He is otherwise healthy.

\section{Does this patient have benign prostatic hyperplasia?}

Men who present to their physician with lower urinary tract symptoms may describe bother from storage (irritative) or voiding (obstructive) symptoms. ${ }^{1}$ Storage symptoms include urinary urgency, frequency or nocturia (nighttime frequency). ${ }^{1}$ Voiding symptoms include urinary hesitancy, weak stream, straining or prolonged voiding. ${ }^{1}$ These symptoms are classically associated with benign prostatic hyperplasia (BPH). A differential diagnosis of urinary tract infection, prostatitis, bladder cancer or urethral stricture may be considered during evaluation with history, physical examination and investigations (see the following sections). A urinary tract infection will likely become apparent with urinalysis and culture; prostatitis with history (chronic symptoms of pain and lower urinary tract symptoms) and examination (tender prostate on digital rectal examination); and bladder cancer (urine cytology) and stricture with further investigation and referral to a urologist.

\section{What questions should this patient be asked on history?}

Medical history should assess the severity of lower urinary tract symptoms and how much the patient is affected by them. Medical comorbidities that may relate to bladder dysfunction include diabetes, neurologic conditions (i.e., Parkinson disease, multiple sclerosis, stroke), previous surgery, trauma or past prostate disease. ${ }^{2}$ All prescription, over-the-counter and herbal medications should be reviewed. Use of the mnemonic FUN WISE (frequency, urgency, nocturia, weak stream, intermittency, straining, emptying incompletely) may aid in remembering the specific assessment of lower urinary tract symptoms. Additional questions should focus on modifiable factors, such as drinking habits, daily fluid intake and caffeine consumption.

\section{What physical examination manoeuvres should be performed?}

In addition to assessment of hernias and previous surgical scars, abdominal examination can help rule out occult urinary retention if the bladder is not palpable. External genitalia should be assessed for abnormalities, including phimosis, and skin rashes caused by incontinence. Guidelines recommend a digital rectal examination as a mandatory component of the evaluation of any male patient with lower urinary tract symptoms. $^{2,3}$ The digital rectal examination should assess size, symmetry, contour (i.e., nodularity) and tenderness of the prostate, as well as anal sphincter tone, and rectal and skin abnormalities.

\section{What tests should be ordered for this patient?}

Guidelines from the Canadian Urological Association recommend that all men presenting with lower urinary tract symptoms have a urinalysis (routine and microscopic) and culture with sensitivity testing to rule out the presence of blood or infectious causes of the symptoms. ${ }^{3}$ Measurement of serum prostate-specific antigen (PSA) levels may be used as a proxy for prostate size and to determine progression or response to therapy for BPH. However, it does not replace performing a digital rectal examination, which must always be done. Prostate-specific antigen levels may be elevated because of prostate enlargement, urinary tract infection, prostatitis, cancer or normal variability. Although screening for prostate cancer is not part of the routine evaluation for BPH, most men presenting with lower urinary tract symptoms are in the age range for which a conversation about prostate cancer screening is recommended. Guidelines from both the Canadian and American urological associations recommend measurement of serum PSA among men with at least a 10-year life expectancy and in whom management would change if prostate cancer were to be detected., ${ }^{2,3}$
Competing interests: None declared.

This article has been peer reviewed.

Correspondence to: Dean Elterman, dean.elterman@uhn.ca

CMAJ 2014. DOI:10.1503 /cmaj.130449 
The Canadian guidelines recommend use of an objective, validated symptom questionnaire because it provides a measure of severity and bother. $^{3}$ The American Urological Association Symptom Index (AUA-SI) is a widely used questionnaire to assess for baseline symptom severity and follow-up symptom evolution. ${ }^{4} \mathrm{~A}$ voiding diary (24-72 hr) will provide additional information on frequency and volume of voiding. ${ }^{3}$ Urine cytology may be done for patients with predominantly storage symptoms, hematuria or risk factors for bladder cancer. ${ }^{3}$ Invasive tests such as cystoscopy and urodynamics, as well as imaging such as abdominal or transrectal ultrasonography, are not recommended in the routine evaluation of lower urinary tract symptoms in men. See Box 1 for a summary of evaluation. ${ }^{3}$

\section{What treatment should be instituted?}

Treatment should be personalized based on symptom severity, degree of bother and patient preference. Behaviour modification should be discussed with patients. These changes include avoidance of aggravating substances such as caffeine, alcohol and excess fluid consumption. Certain medications, such as diuretics, may be adjusted in dose or timing. Fluid restriction before bedtime may help with symptoms of nocturia. ${ }^{3}$ In our opinion, patients with moderate to severe symptoms or whose symptoms do not respond to six to eight weeks of lifestyle interventions may either receive first-line medical therapy from the primary care physician or be referred to a urologist.

First-line medical therapies for bothersome symptoms of the lower urinary tract are $\alpha$-blockers (i.e., doxazosin, tamsulosin, terazosin, alfuzosin and silodosin). ${ }^{2,3}$ A meta-analysis showed that all agents in this class are fairly equivalent in terms of efficacy, with individual agents differing slightly in adverse effect profile. ${ }^{4}$ Possible adverse effects of these agents include hypotension $(5 \%-10 \%)$, postural or orthostatic hypotension
(5\%-10\%), dizziness $(5 \%-10 \%)$, headache $(5 \%)$, asthenia (5\%), nasal congestion $(5 \%)$ and retrograde ejaculation $(3 \%-10 \%) .{ }^{5}$ Men can expect to see improvement in symptoms about three to six days following initiation of an $\alpha$ blocker. We believe that symptom improvement should be assessed four to six weeks after initiation of $\alpha$-blocker therapy, and measured either as subjective improvement in symptoms or using a validated measurement tool, such as the AUA-SI., ${ }^{2,6}$

The $5 \alpha$-reductase inhibitors (5-ARIs) (i.e., finasteride and dutasteride) may be given alone or in combination with an $\alpha$-blocker in men with enlarged prostates. ${ }^{7.8}$ These medications reduce prostate volume, thereby improving symptoms, and reducing the risk of acute urinary retention and need for surgery. ${ }^{7}$ Serum PSA should be measured at baseline before the start of 5-ARI therapy. Possible adverse effects of these agents include erectile dysfunction, decreased libido, decreased ejaculate volume, and breast tenderness or enlargement. Treatment with 5-ARIs typically takes six months before symptomatic improvement and an expected 50\% reduction in serum PSA level. ${ }^{9}$

In men with comorbid $\mathrm{BPH}$ and erectile dysfunction, phosphodiesterase-5 (PDE-5) inhibitors (e.g., tadalafil, sildenafil and vardenafil) have been shown to improve lower urinary tract symptoms secondary to $\mathrm{BPH} .{ }^{10}$ Only tadalafil $5 \mathrm{mg}$ daily is approved in Canada and the United States for the treatment of both erectile dysfunction and BPH. Possible adverse effects of PDE-5 inhibitors include headache, dyspepsia, facial flushing, nasopharyngitis and backache. ${ }^{11}$

Phytotherapies, such as saw palmetto, are not recommended for use in $\mathrm{BPH}$, because no difference compared with placebo has been shown in systematic reviews of the literature. ${ }^{2,12,13}$

Pharmacotherapy should be maintained indefinitely in patients who experience improved symptoms.

\begin{tabular}{|c|c|c|c|}
\hline Evaluation & Mandatory in every patient & Optional & Not recommended \\
\hline History & $\begin{array}{l}\text { - General medical } \\
\text { - Focused urologic history }\end{array}$ & Voiding diary & Not applicable \\
\hline $\begin{array}{l}\text { Physical } \\
\text { examination }\end{array}$ & $\begin{array}{l}\text { - General physical } \\
\text { - Focused urologic, including } \\
\text { digital rectal, examination }\end{array}$ & $\begin{array}{l}\text { - Uroflow test } \\
\text { - Postvoid residual urine } \\
\text { test }\end{array}$ & Cystoscopy \\
\hline $\begin{array}{l}\text { Ancillary } \\
\text { tests }\end{array}$ & $\begin{array}{l}\text { Urinalysis (routine and } \\
\text { microscopic, and culture and } \\
\text { sensitivity) }\end{array}$ & $\begin{array}{l}\text { - Symptom inventory } \\
\text { - Prostate-specific antigen } \\
\text { test (if indicated) } \\
\text { - Serum creatinine test } \\
\text { - Urine cytology (if storage } \\
\text { symptoms) }\end{array}$ & $\begin{array}{l}\text { - Ultrasonography or } \\
\text { imaging of prostate } \\
\text { or upper urinary } \\
\text { tracts } \\
\text { - Urodynamic studies }\end{array}$ \\
\hline
\end{tabular}




\section{When should this patient be referred to a urologist?}

As mentioned, we believe that men with moderate to severe symptoms or whose symptoms did not respond to six to eight weeks of lifestyle interventions may be referred to a urologist for further evaluation or initiation of medical management. ${ }^{6}$ Patients with complicated symptoms, including microscopic or gross hematuria, recurrent urinary tract infections, urinary retention, renal insufficiency from obstruction, prior urologic surgery or persistent symptoms should be referred for urologic assessment. ${ }^{3,6}$ Rising PSA levels, especially during 5-ARI treatment, or a suspicion of prostate cancer based on history, digital rectal examination or serum PSA level also warrants referral to a urologist. Surgical intervention is reserved for patients with moderate to severe symptoms whose symptoms do not respond to, or who do not want, medical therapy. Minimally invasive transurethral resection of the prostate remains the gold standard for surgical therapy for $\mathrm{BPH} .{ }^{3}$ However, various technologies using different forms of energy to resect, ablate or vaporize prostate tissue are available and include mono- and bipolar cautery loop, GreenLight (potassium titanyl phosphate [KTP]) laser and holmium laser. ${ }^{3}$

\section{Case resolution}

Our patient's symptoms (urinary urgency, nocturia and slow stream) were evaluated according to the recommendations summarized in Box 1. After the history, physical examination and investigation, he started a trial period of behavioural modification. If, after six to eight weeks, his symptoms persist, initiation of firstline medical therapy with an $\alpha$-blocker would be the next step. If his prostate had been enlarged or if he had been experiencing erectile dysfunction, adding a 5-ARI or PDE-5 inhibitor, respectively, would have been appropriate. In the event of no improvement in lower urinary tract symptoms or complicated symptoms (as outlined in the previous section), the patient should be referred to a urologist.

\section{References}

1. Abrams P, Cardozo L, Fall M, et al. The standardisation of terminology of lower urinary tract function: report from the Standardisation Sub-committee of the International Continence Society. Neurourol Urodyn 2002;21:167-78.

2. McVary KT, Roehrborn CG, Avins AL, et al. Update on AUA guideline on the management of benign prostatic hyperplasia. J Urol 2011;185:1793-803.

3. Nickel JC, Méndez-Probst CE, Whelan TF, et al. 2010 update: guidelines for the management of benign prostatic hyperplasia. Can Urol Assoc J. 2010;4:310-6.

4. Marberger M, Harkaway R, de la Rosette J. Optimising the medical management of benign prostatic hyperplasia. Eur Urol 2004; 45:411-9.

5. Milani S, Djavan B. Lower urinary tract symptoms suggestive of benign prostatic hyperplasia: latest update on alpha-adrenoceptor antagonists. BJU Int 2005;95(Suppl 4):29-36.

6. Abrams P, Chapple C, Khoury S, et al. Evaluation and treatment of lower urinary tract symptoms in older men. J Urol 2009;181: 1779-87.

7. McConnell JD, Roehrborn CG, Bautista OM, et al. The longterm effect of doxazosin, finasteride, and combination therapy on the clinical progression of benign prostatic hyperplasia. N Engl J Med. 2003;349:2387-98.

8. Toren P, Margel D, Kulkarni G, et al. Effect of dutasteride on clinical progression of benign prostatic hyperplasia in asymptomatic men with enlarged prostate: a post hoc analysis of the REDUCE study. BMJ 2013;346:f2109.

9. Azzouni F, Mohler J. Role of 5 $\alpha$-reductase inhibitors in benign prostatic diseases. Prostate Cancer Prostatic Dis 2012;15:222-30.

10. Wong P, Lawrentschuk N, Bolton DM. Phosphodiesterase 5 inhibitors in the management of benign prostatic hyperplasia and erectile dysfunction: the best of both worlds. Curr Opin Urol 2009; 19:7-12.

11. Miller MS. Role of phosphodiesterase type 5 inhibitors for lower urinary tract symptoms. Ann Pharmacother 2013;47:278-83.

12. Tacklind J, Macdonald R, Rutks I, et al. Serenoa repens for benign prostatic hyperplasia. Cochrane Database Syst Rev 2012; 12:CD001423.

13. Barry MJ, Meleth S, Lee JY, et al. Effect of increasing doses of saw palmetto extract on lower urinary tract symptoms: a randomized trial. JAMA 2011;306:1344-51.

Affiliations: Division of Urology (Elterman), University Health Network, University of Toronto, Toronto, Ont.; James Buchanan Brady Foundation, Department of Urology (Kaplan), Iris Cantor Men's Health Center, Weill Cornell Medical College of Cornell University, New York, NY

Contributors: Both authors drafted and revised the article, and approved the version submitted for publication.

Decisions is a series that focuses on practical evidence-based approaches to common presentations in primary care. The articles address key decisions that a clinician may encounter during initial assessment. The information presented can usually be covered in a typical primary care appointment. Articles should be no longer than 650 words, may include one box, figure or table and should begin with a very brief description ( 75 words or less) of the clinical situation. The decisions addressed should be presented in the form of questions. A box providing helpful resources for the patient or physician is encouraged. 